\title{
Progression of Pseudolymphoma to true Cutaneous lymphoma, possible role of Platelet-rich plasma (PRP), a case report.
}

Kamran Balighi ${ }^{1}$, martin kasir ${ }^{2}$, maryam vahedi ${ }^{3}$, Alireza Ghanadan ${ }^{3}$, and Nasim Tootoonchi ${ }^{3}$

${ }^{1}$ TUMS

${ }^{2}$ Dallas ISD

${ }^{3}$ Tehran University of Medical Sciences

November 23, 2021

\begin{abstract}
here we report a case of cutaneous Pseudolymphoma progression to frank cutaneous lymphoma and resistant to conventional therapy after receiving Platelet-rich plasma and hair transplantation .these modalities and their proliferative formulation may be the leading cause or aggravating factor for such transformation's specially for patients with positive family history,
\end{abstract}

Title: Progression of Pseudolymphoma to true Cutaneous lymphoma, possible role of Plateletrich plasma (PRP), a case report.

Running Title: Progression to lymphoma after

Platelet-rich plasma (PRP).

Kamran Balighi ${ }^{1}$ MD, Martin Kassir ${ }^{2}$ MD,Maryam Vahedi Namin ${ }^{1}$ MD,Alireza Ghanadan ${ }^{3}$, Nasim Tootoonchi ${ }^{1}$ MD.

1. Autoimmune Bullous Diseases Research Center, Razi Hospital, Tehran University of medical sciences, Tehran, Iran.

2. Worldwide laser institute, Dallas, TX, USA

3. Dermatopathology department, Razi Hospital, Tehran University of Medical Sciences,Tehran,Iran.

* Corresponding author: Nasim Tootoonchi, Department of Dermatology, Razi Hospital, Tehran University of Medical Sciences, Tehran, Iran.

Razi Hospital- Vahdate Eslami Street- Tehran- Iran

Zip code: 1199663911 Tel: 00982155618989

Email address: doctor.tootoonchi@gmail.com

- Manuscript word count: 924

- Reference count:7

- Figures:2

Acknowledgment: This research has been supported by Tehran University of medical sciences.

Conflicts of Interest : No relevant conflicts of interest to disclose.The study protocol was approved by the ethics committee of Tehran University of Medical Sciences . 


\section{Authors Contribution Statement}

Kamran balighi: conception and design, revising the manuscript, Given final approval Martin Kassir: conception and design, revising the manuscript, Maryam Vahedi Namin: acquisition of data, drafting the manuscript. Alireza Ghanadan: acquisition of data. Nasim Tootoonchi: drafting the manuscript, analysis and interpretation of data.

- Funding information: No funding sources

- Written informed consent was obtained from the patient to publish this report in accordance with the journals patients consent policy.

- Data available on request from the authors .

Title: Progression of Pseudolymphoma to true Cutaneous lymphoma, possible role ofPlateletrich plasma (PRP), a case report.

\section{Running Title: Progression to lymphoma after \\ Platelet-rich plasma (PRP).}

Keywords : cutaneous pseudolymphoma. Primary cutaneous lymphomas. Platelet-rich plasma (PRP).

Introduction:

Cutaneous pseudolymphoma (CPL), also called cutaneous lymphoid hyperplasia, is a nonspecific disease that is characterized by a polyclonal benign lymphoproliferative process ${ }^{1,2}$. Pseudolymphoma can mimic lymphoma both clinically and histologically. ${ }^{1.2 .3 .4}$ Most cases are idiopathic. Known etiologies include reaction to tattoo, arthropod bite, infection (borrelia burgdorferi, molluscum contagiosum ${ }^{2}$, leishmaniasis), vaccination and drugs (e.g., anticonvulsants).

Primary cutaneous lymphomas are a heterogenous group of lymphoproliferative disorders that primarily involve the skin. They are the most frequent extra nodal lymphomas; classification is based on lymphocyte type and includes B-cell and T-cell. Cutaneous T-cell lymphoma (CTCL) is the most common type. ${ }^{6}$. Cutaneous B-cell lymphomas are classified into four types: 1) primary cutaneous marginal zone B-cell lymphoma (PCMZL) 2) primary cutaneous follicular center lymphoma (PCFCL) 3) primary cutaneous diffuse large B-cell lymphoma, leg type ( LBCL-L) 4) primary cutaneous large B-cell lymphoma, other(LBCLother $)^{7,8}$

Platelet-rich plasma (PRP) is a biological product defined as a portion of the plasma fraction of autologous blood with a platelet concentration above the baseline. It is obtained from the blood of patients collected before centrifugation. Platelets contain growth factors and mediators in their granules (TGF-B1, PDGF, bFGF, VEGF, EGF, IGF-1) and believed to promote cell proliferation, differentiation, angiogenesis and chemotaxis. PRP is considered a novel treatment modality especially for hair restoration and skin rejuvenation, in combination with other modalities or alone. ${ }^{9}$

Case presentation:

A 46-year-old man was referred to our hospital with complaint of erythematous plaques on his head, especially the forehead, that started 20 years ago. Over time, the lesions had increased in size. the patient had no remarkable past medical history or drug history. In 2009, a skin biopsy was performed showing cutaneous B-cell pseudolymphoma (CBPL). Immunohistochemistry (IHC) performed in the same year revealed CD20, CD3, CD4, CD8, CD79a and BCL2 were positive and, no evidence of lymphomatous involvement and no evidence of clonal staining for Kappa and Lambda light chains. The patient was lost to follow up and treatment until 2013, at which time repeat biopsy was performed and a diagnosis of pseudolymphoma with dense lymphoid infiltration with germinal center follicles was confirmed. After diagnosis, patient received 12 sessions of monthly intralesional corticosteroids. Following treatment, the patient's lesions improved significantly and almost resolved. In 2017, the patient had a hair transplant followed by a platelet-rich plasma (PRP) session. One week after PRP, Following the first session of PRP, lesions re-appeared on the 
head (FIGURE 1A). Re-biopsy from one of the new lesions once again showed pseudolymphoma. The patient was re-treated with intralesional corticosteroids and topical clobetasol, but was not responsive to treatment. The patient also did not respond to treatment with hydroxychloroquin and thalidomide.following enlargement of the previous lesions, the biopsy was repeated, and atypical nodular and diffuse lymphoid cell infiltration was seen, compatible with lymphoproliferative disorder (FIGURE2A) and IHC study recommended. In IHC, the finding (CD20 strongly positive and BCL2 and BCL6 positive) was in favor of primary cutaneous follicular center lymphoma (PCFCL) (FIGURE 2 B,C,D,E). The patient did not have lymphadenopathy on examination and paraclinical tests were normal. Also, chest, neck and abdominal and pelvic CT scan were normal, consistent with being primary cutaneous origin lymphoma. in the meantime, positive family history for Hodgkin lymphoma in patient's sister revealed. Due to the lack of response to previous treatment, the patient became candidate for rituximab (6-8 sessions every three weeks). At present, the patient has had a significant improvement following receiving rituximab. (FIGURE 1 B)

Discussion:

In this study, a rare manner of cutaneous pseudolymphoma (CPL)with non-ordinary progression time line that advanced into primary cutaneous follicular center lymphoma is presented. This patient was confirmed case of cutaneous B-cell pseudolymphoma (CBPL)for almost 4 years and had complete response following intralesional corticosteroid therapy. But just after hair transplantation and PRP sessions the disease recured, and at this time following histopathology and immunohistochemistry the primary follicular center B cell lymphoma was diagnosed. For us it was an unanswered question that if these lesions represent actual progression from cutaneous pseudo lymphoma to frank lymphoma as it has been previously discussed in literature ${ }^{5}$ and also Kulow et al reported a 4 patients initially diagnosed with combination of clinical, histopathology and immunophenotyping as cutaneous pseoudolymphoma and had a progressive clinical course evolving as primary cutaneous lymphoma.${ }^{4}$ or they were malignant from beginning and not diagnosed properly which it seems unlikely due to long duration of disease and complete response and clearance after intralesional steroid therapy.

On the other hand, PCFCL is considered as the most common cutaneous lymphoma ${ }^{12}$. While the clear pathogenesis of PCFCL has remained unknown, Persistent antigenic stimulation has been suggested as a cause for such a progression ${ }^{11}$. Thus, a long-time follow-up is also absolutely necessary to make the final diagnosis.

In this study our case underwent various interventions including hair transplantation and PRP. PRP consist of growth factors and hormones which potentially could lead to excess cell proliferation. It is unknown that these modalities alone or their specific proliferative formulations may be the leading cause or aggravating factor ending to primary cutaneous lymphoma specially for patients with positive family history. According to recent reports ${ }^{12}$ and formation of malignancies after PRP ,it seems wise to be cautious specially for patients with positive family or personal history and further research should be done to investigate the possibility and association of these factors.

*Acknowledgment:

This research has been supported by Tehran University of medical sciences.

References:

1. Mitteldorf C, Kempf W. Cutaneous pseudolymphoma-A review on the spectrum and a proposal for a new classification. J Cutan Pathol. 2020 Jan;47(1):76-97. doi: 10.1111/cup.13532. Epub 2019 Jul 31. PMID: 31237707 .

2. Shalabi D, Ringe J, Lee JB, Nikbakht N. Molluscum contagiosum infection with features of primary cutaneous anaplastic large cell lymphoma. Dermatol Online J. 2019 Nov 15;25(11):13030/qt7vp863hz. PMID: 32045148.

3. Tian Z, Shiyu Z, Tao W, Li L, Yuehua L, Hongzhong J. Lymphoma or pseudolymphoma: A report of six 
cases and review of the literature. Dermatologic Therapy. 2019;32: e12807.

4.. Kulow BF, Cualing H, Steele P, VanHorn J, Breneman JC, Mutasim DF, Breneman DL. Progression of cutaneous B-cell pseudolymphoma to cutaneous B-cell lymphoma. J Cutan Med Surg. 2002 NovDec;6(6):519-28. doi: 10.1007/s10227-001-0133-7. Epub 2002 Oct 31. PMID: 12404039

5. Knowles 2nd DM, Halper JP, Jakobiec FA. The immunologic

characterization of 40 extranodal lymphoid infiltrates: usefulness

indistinguishing between benign pseudolymphoma and malignant

lymphoma. Cancer 1982; 49:2321-2335

6. Nicolay JP, Wobser M. Cutaneous B-cell lymphomas - pathogenesis, diagnostic workup, and therapy. J Dtsch Dermatol Ges. 2016 Dec;14(12):1207-1224. doi: 10.1111/ddg.13164. PMID: 27992127.

7. Grandi V, Alberti Violetti S, La Selva R, Cicchelli S, Delfino C, Fava P, Fierro MT, Pileri A, Pimpinelli N, Quaglino P, Berti E. Primary cutaneous B-cell lymphoma: narrative review of the literature. G Ital Dermatol Venereol. 2019 Aug;154(4):466-479. doi: 10.23736/S0392-0488.17.05670-X. Epub 2017 Nov 16. PMID: 29144099

8. Willemze R, Jaffe ES, Burg G, et al. WHO-EORTC classification for cutaneous lymphomas. Blood. 2005;105(10):3768-3785. doi:10.1182/blood-2004-09-3502

9. Emer J. Platelet-Rich Plasma (PRP): Current Applications in Dermatology. Skin Therapy Lett. 2019;24(5):1-6.

10. Skala SL, Hristov B, Hristov AC. Primary Cutaneous Follicle Center Lymphoma. Arch Pathol Lab Med. 2018;142(11):1313-1321. doi:10.5858/arpa.2018-0215-RA

11Breza TS Jr, Zheng P, Porcu P, Magro CM. Cutaneous marginal zone B-cell lymphoma in the setting of fluoxetine therapy: a hypothesis regarding pathogenesis based on in vitro suppression of T-cell-proliferative response. J Cutan Pathol. 2006;33(7):522-528. doi:10.1111/j.1600-0560.2006.00475.x.

12. Jean-Noël Dauendorffer, Sylvie Fraitag, Alain Dupuy. Carcinome basocellulaire après " réjuvénation " par injection de plasma autologue riche en plaquettes / Basal cell carcinoma following platelet-rich plasma injection for skin rejuvenation. Annales de Dermatologie et de Vénéréologie, Elsevier Masson, 2013, 140 (11), pp.723-4. ¡10.1016/j.annder.2013.07.010i.

Figure legends:

Figure 1:

(A)Erythematous and indurated plaques and nodules on scalp, forehead extending to vertex

(B) significant resolution of lesions after rituximab therapy.

Figure 2:

Primary cutaneous follicle center lymphoma, diffuse type (A-E).

(A)Diffuse and nodular growth pattern infiltrate of large lymphoid cells with lack of mantle zone and tinigible body macrophages

(B)CD 10+ . (C) Bcl-6+. (D) CD 20+. (E) CD23+ meshwork pattern. 


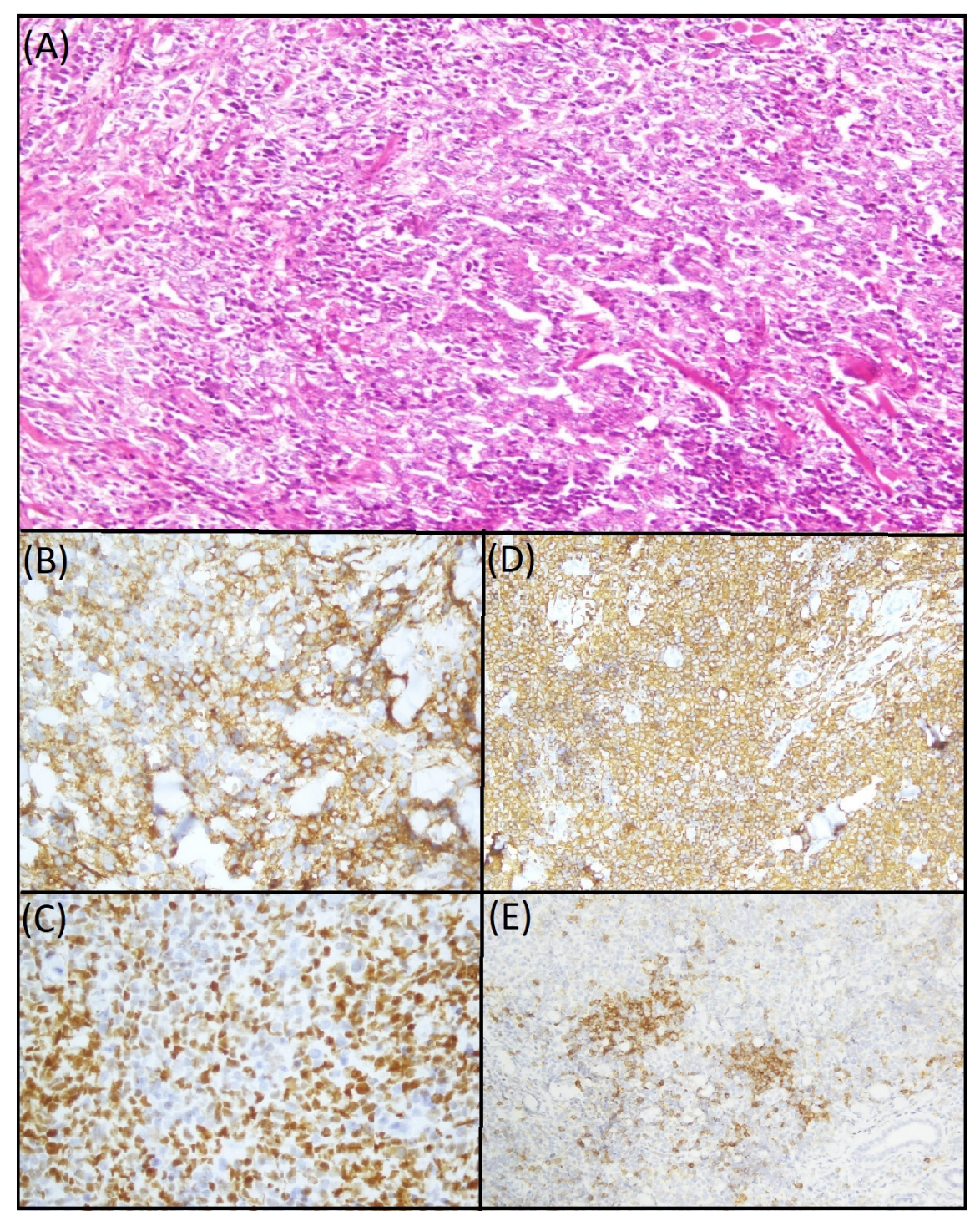




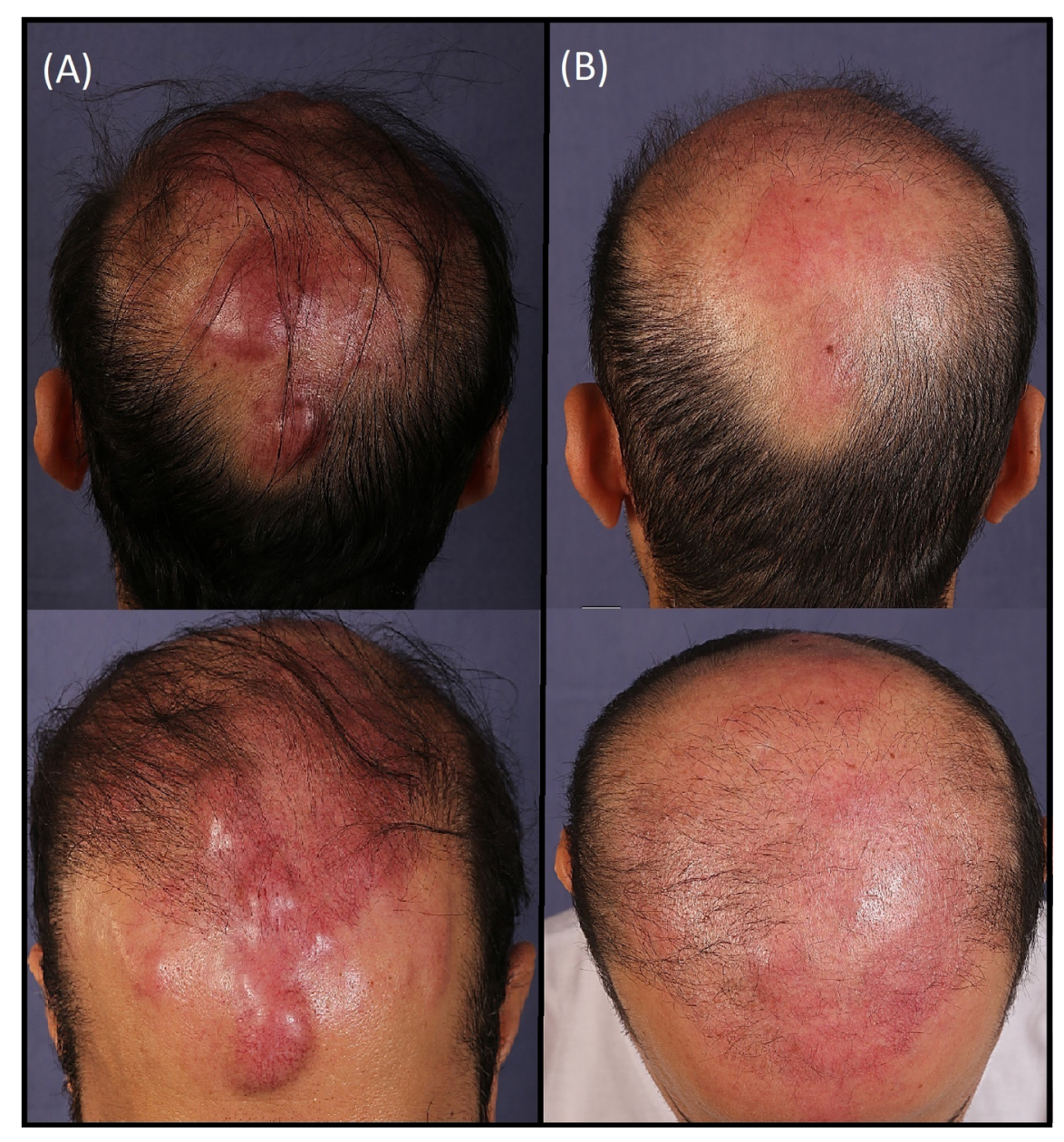

